\title{
Natural products and evolutionary ecology
}

\author{
Otto R. Gottlieb and Maria Renata de M. B. Borin \\ Departamento de Fisiologia e Farmacodinâmica, Instituto Oswaldo Cruz, \\ FIOCRUZ, 21045-900 Rio de Janeiro, RJ, Brasil
}

\begin{abstract}
Quantitative phytochemistry, a novel method incorporating data on metabolism, morphology and ecogeography, is applied to the calculation of a phylogenetic scheme. This indicates angiosperm evolution to proceed by alternation of order and chaos. The transition of order into chaos is attributed to the interaction of antagonistic forces of similar potency (evolution). The transition of chaos into order is attributed to the selective action of an environmental constraint such as atmospheric oxygen (ecology). The observations are postulated to possess general validity and to explain evolutionary mechanisms such as gradualism and punctuation.
\end{abstract}

\section{INTRODUCTION}

Plants must be by far the largest group of living organisms in which it is still necessary to specify on each occasion the particular phylogenetic arrangement to be followed (1). Comparative analysis of the major modern versions of such arrangements (2) shows that, in contrast with most other groups of organisms, the evolution of angiosperms cannot be adequately expressed by existing, chiefly morphological, evidence in directional cladograms (3). It is the purpose of the present work to clarify the reasons of this fact and to disclose the evolutionary laws underlying angiosperm macrophylogeny. To this end experimental findings concerning metabolism-morphology-ecogeography (4) are evaluated.

\section{RESULTS}

Dynamical evolutionary trends are easiest to perceive through the examination of metabolic gradients, such as, in angiosperms, the stepwise curtailment of the shikimate pathway via evolutionary canalization (5) and the progressive increase of the mean oxidation level of compounds within particular metabolic categories (6). These metabolic transitions coincide in first approximation with the separation of the magnolialean and the rosiflorean blocks [(sensu Kubitzki and Gottlieb (3)]. Oxidative shikimate and acetate based pathways mark respectively the subclasses Magnoliidae and Asteridae [sensu Cronquist (7)], while non-oxidative mixed pathways uniformly characterize the subclasses Hamamelidae, Dilleniidae and Rosidae, here designated the HDR-complex (Table 1). These qualitative deductions were quantified by classification of families either according to herbaceousness indices (HI $=100-$ WI, i.e. woodiness index) (8) and shikimate/acetate proportion [data base (4)] (metabolism), Sporne indices (SI) (9) (morphology), as well as habitats $(10,11)$ (ecogeography).

Metabolism, as measured by lignin deposition (WI) indicated by HI (Fig. 1), reveals Ranunculidae and Caryophyllidae to be late branches of the magnolialean block. The HDR-complex is characterized by strengthening of lignification. Gallic acid, a known activator of PAL (12) responsible for this effect, should appear by evolutionary canalization of the shikimate pathway and act through a feedback mechanism. In Rosidae, caffeic acid, a known inhibitor of PAL (12), antagonizes gallic acid. Hence this subclass approaches Asteridae in distribution of woody families. In Asteridae these families may be either transitional or secondarily woody since the trend towards herbaceousness here is very conspicuous.

Metabolism, as measured by the percentage of shikimate derivatives (SH), was postulated to represent the relative potency of the lignin attractor. The calculated values (Fig. 2) show this to be strongest for Ranunculidae (97.4\%) and Magnoliidae (94.3\%) and weakest for Caryophyllidae $(26.1 \%)$ and 
Asteridae (21.3\%). Not surprisingly Hamamelidae (71.5\%), Dilleniidae (71.3\%) and Rosidae (68.0\%) assume intermediate positions. Located close to the critical (dashed) line ( $\mathrm{SH}=\mathrm{AC}=50 \%$ ) where the interchange of woody and herbaceous forms should be easiest, they must lack uniquely defined features and occupy uncertain positions in classificatory schemes. Thus, the HDR-complex is more variable (and hence adaptable) than the remaining subclasses.

TABLE 1. Characteristics of angiosperm subclasses. Magnoliidae, Ranunculidae and Caryophyllidae (Mag), Hamamelidae, Dilleniidae and Rosidae (HDR) and Asteridae (Ast)

\begin{tabular}{clll}
\multicolumn{1}{c}{ Characteristics } & \multicolumn{1}{r}{ Mag } & \multicolumn{1}{c}{ ADR } & \multicolumn{1}{c}{ Ast } \\
\hline Metabolism - & shikimate & shikimate/acetate & acetate \\
Categories & few & many & few \\
Alkaloids & frequent & rare & frequent \\
Polyphenols & rare & frequent & rare \\
Evolutionary gradualism & oxidative & absent & oxidative \\
Morphology - Habit & woody & shrubby & herbaceous \\
$\mathrm{N}^{2}$ of species per family & 539 & $409^{*}$ & 1,229 \\
Ecogeography - Habitat & tropical & variable & temperate \\
Distribution & restrict & vast & restrict \\
\hline
\end{tabular}

* HDR without Fabales $=336$.
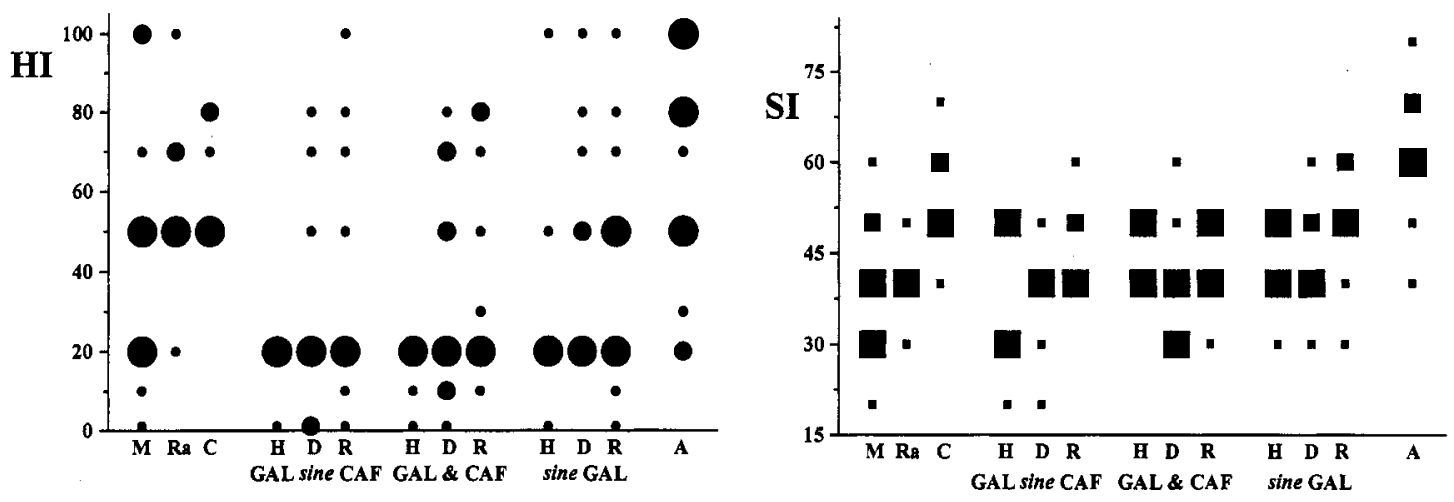

Fig. 1 Relative importance of families of angiosperm subclasses characterized by herbaceousness indices (HI) and Sporne indices (SI). Magnoliidae (M), Ranunculidae (Ra), Caryophyllidae (C) and Asteridae (A). Hamamelidae (H), Dilleniidae (D) and Rosidae (R) are separated according to presence and absence of gallo- (GAL) and caffeoyl- (CAF) tannins.

Morphology, as measured by SI (Fig. 1), again reveals the most primitive Magnoliidae (SI $=30$ for 6 families) to be followed by Ranunculidae (SI $=40$ for 5 families) and Caryophyllidae (SI $=50$ for 4 families), while Asteridae (SI $=60$ for 21 families) appear as the most modern outcrop. The HDR-complex is characterized by intermediate Sporne indices with low variation. Additionally the two subclasses, Magnoliidae and Ranunculidade (SI $=40$ for 10 families) lead via Rosidae sine gallic acid (SI $=50$ for 13 families) towards Asteridae ( $\mathrm{SI}=60$ for 21 families).

Comparison of the HI-oriented data with the SI-oriented data (Fig. 1) reveals habit (lignification) to be guided less by evolutionary genetics than by evolutionary ecology (i.e. by atmospheric oxygen pressure). The ecological nature of this phenomenon is confirmed by the intimate dependence of atmospheric oxygen content and extent of lignified forms throughout the Phanerozoic (11).

Ecogeography. In contrast to all other data of the present work, which refer to world-wide profiles (4), data of this section refer to 80 regions located in South America (10). Families of Magnoliidae predominate in forests, but lose importance gradually towards steppes (Table 2). Families of Ranunculidae, of Caryophyllidae and very conspicuously of Asteridae increase countercurrently. In contrast to the irregular habitat-dependent distribution of the number of Magnoliidae and Asteridae 
families, the analogous distribution of families belonging to each of the subclasses Hamamelidae, Dilleniidae and Rosidae is strikingly uniform, latitudinal trends not being observable.

Thus HDR maintain a singular lack of order, extreme chemical plasticity allowing their penetration into very diverse habitats (Table 1,2), including regions of transition between two or more diverse communities. Such ecotones are generally characterized by high biological diversity due to intense bioticabiotic interactions (13).

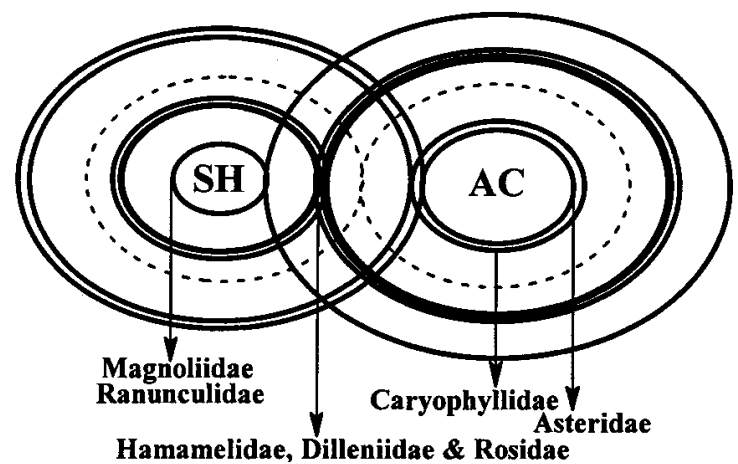

Fig. 2 Antagonism of metabolic attractors, shikimate (SH) versus acetate (AC), for angiosperm subclasses.

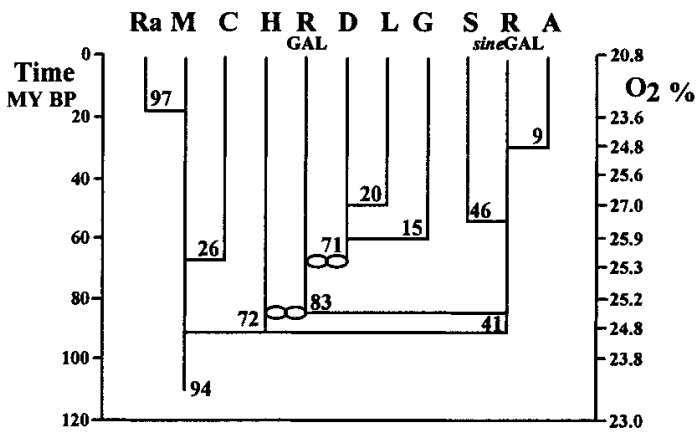

Fig. 3 Phylogenetics of dicotyledons. Atmospheric oxygen and shikimate derivatives $(\mathrm{SH})$ at time of origin of groups.

TABLE 2. Mean percentages of families of angiosperm subclasses classified according to types of vegetation and range of latitudes, based on 80 South America floristic inventories

\begin{tabular}{|c|c|c|c|c|c|c|c|c|c|c|c|c|c|c|}
\hline \multirow[b]{2}{*}{ Vegetation } & \multirow{2}{*}{$\begin{array}{c}\text { Latitude } \\
\left({ }^{\circ} \mathrm{S}\right)\end{array}$} & \multirow[b]{2}{*}{ Mag } & \multirow[b]{2}{*}{ Ran } & \multirow[b]{2}{*}{ Car } & \multicolumn{3}{|c|}{ GAL sine CAF } & \multicolumn{3}{|c|}{ GAL \& CAF } & \multicolumn{3}{|c|}{ sine GAL } & \multirow[b]{2}{*}{ Ast } \\
\hline & & & & & Ham & Dil & Ros & Ham & Dil & Ros & Ham & Dil & Ros & \\
\hline Upland For & $2-12$ & 8 & 2 & 2 & - & 6 & 11 & 2 & 9 & 20 & 2 & 10 & 6 & 10 \\
\hline Cerrados & $13-22$ & 5 & 2 & 3 & - & 8 & 9 & 1 & 6 & 22 & 2 & 7 & 3 & 17 \\
\hline Open Fields & $23-30$ & 4 & 3 & 6 & - & 5 & 10 & 1 & 9 & 17 & 2 & 6 & 5 & 24 \\
\hline Steppes & $40-46$ & 2 & 4 & 6 & - & 2 & 6 & 2 & 6 & 18 & 2 & 7 & 5 & 24 \\
\hline
\end{tabular}

\section{DISCUSSION}

At this point of the argument, the phylogenetic relationships of the angiosperms have been recognized to involve three major trends. These operate in the basal Magnoliidae with evolutionary lineages marked by neolignans and benzylisoquinoline alkaloids (further diversified in Ranunculidae and Caryophyllidae), in the intermediate HDR-complex (except Rosidae sine gallic acid) with strikingly disjunct characteristics, and in the Rosidae sine gallic acid and the climactic Asteridae with renewal of gradual evolutionary developments.

A plot of these data (Fig. 3) discloses some hitherto unsuspected relationships. According to paleopalinologic data (14), the first bifurcation of the basal Magnoliopsid lineage leads alternatively to Ranunculidae-Caryophyllidae, or to Hamamelidae and Rosidae. The latter subclass splits in two branches, one continuing exempt of gallic acid and another one deriving gallic acid possibly by genetic compatibility with Hamamelidae in a region of ecological stress. Gradual loss of gallic acid, caused by the antagonistic action of caffeic acid, leads to Dilleniidae. According to data on atmospheric oxygen content (15), development of the HDR-complex coincided with increase of oxygen pressure which attained the maximum at the time of separation of Gentiananae and Lamianae, two superorders [sensu Dahlgren (16)] of Asteridae. In parallel the gallic acid free branch of Rosidae evolved into Solananae and Asteranae, a demonstration that Cronquist's subclass Asteridae must be polyphyletic.

Thus, in the primitive magnolialean block evolutionary gradualism is established by oxidative sequences involving shikimate derivatives (high $\mathrm{SH}$ ). Order in the recent part of the rosiflorean block is renewed by 
oxidative sequences based on acetate derivatives (low $\mathrm{SH}$ ). The crucial point of this scheme involves the bifurcation of the Rosidae with a shikimate percentage of 41 , i.e. near the equilibrium situation of $50 \%$. At this point metabolic regulation of plant life is attenuated. Consequently, in analogy with chaotic systems, evolution in the remote part of the rosiflorean block becomes strongly dependent on chance and hence endowed with maximal creative potential. Here, characters of metabolic or other nature lack usefulness as phyletic or classificatory markers. Furthermore, SH-values for the gallic acid containing lineages $[72,83,71]$ tend to be closer to the critical $\mathrm{SH}=50$ than for the gallic acid free ones $[94,26,97$; $20,15$ and $41,46,9]$. Thus, rate of evolution should be faster in the former chaotic situation than in the latter cases of phyletic gradualism (Fig. 3).

For any environmental feature to perform the role of "Maxwell's demon" (17) and renew order from chaos, it must be able to interact with plant constituents. Oxygen is certainly the most likely agent for this action. Indeed the major episodes of establishment of order within the angiosperms, their origin and their development into Gentiananae and Lamianae, coincided respectively with an increase and a maximum of atmospheric oxygen content. This fact constitutes additional evidence for the influence of oxygen pressure in species diversity of all plant divisions (6).

\section{CONCLUSION}

The quantitative phytochemical evidence used in the construction of the phyletic scheme is based on data obtained for extant angiosperms in their natural habitats, and is complemented by further organizational levels such as morphology and ecogeography. The evolution/ecology based mechanisms observed are thus organocentric and may surpass in significance the more modern genocentric systems. Application to Darwin's phyletic gradualism would be expressed within biosynthetic categories via stepwise oxidative modifications and among biosynthetic categories via stepwise increase or shortening of sequences of metabolic intermediates. Given the dependence on enzymatic systems, evolutionary processes are slow and gradual phenomena. Eldredge and Gould's punctuation would be expressed by metabolic activation/inhibition via alternation of metabolic attractors. Given the dependence on adaptive plasticity, ecological processes are fast and diversificatory phenomena.

Acknowledgements: The authors are indebted to Conselho Nacional de Desenvolvimento Cientifico e Tecnológico for fellowships, to Academia Brasileira de Ciências for a grant and to Dorothea H. T. Zocher, B.Sc., and José Rubens Toledo Barros, B.Sc. for competent assistance in the construction of the data base.

\section{REFERENCES}

1. N. F. Hughes. In Origin and Early Evolution of Angiosperms (C. B. Beck, ed.), pp.11-22. Columbia University Press, New York (1976).

2. A. Goldberg. Classification, Evolution and Phylogeny of the Families of Dicotyledons, Smithsonian Contributions of Botany 58, 314 pp. Smithsonian Institution Press, Washington (1986).

3. K. Kubitzki and O. R. Gottlieb. Taxon 33, 375-391 (1984).

4. O. R. Gottlieb, M. R. de M. B. Borin and M. A. C. Kaplan. Phytochemistry 40, 99-113 (1995).

5. O. R. Gottlieb. Phytochemistry 29, 1715-1724 (1990).

6. O. R. Gottlieb. Phytochemistry 28, 2545-2558 (1989).

7. A. Cronquist. The Evolution and Classification of Flowering Plants, 2nd ed., 555 pp., The New York Botanical Garden, New York (1988).

8. M. R. de M. B. Borin and O. R. Gottlieb. Pl. Syst. Evol. 184, 41-76 (1993).

9. K. R. Sporne. New Phytol. 85, 419-499 (1980).

10. O. R. Gottlieb and M. R. de M. B. Borin. Opera Bot. Belg. 7, 213-230 (1996)

11. O. R. Gottlieb, M. R. de M. B. Borin and B. M. Bosisio. Anais Acad brasil. Ci. 67 (Supl. 3), 355-362 (1995).

12. A. Boudet, R. Ranjeva and P. Gadel. Phytochemistry 10, 997-1005 (1971).

13. M. M. Holland and P. G. Risses. In Ecotones, The Role of Landscape Boundaries in the Management and Restoration of Changing Environments (M. M. Holland, P. G. Risses and R. J. Naiman, eds.), pp.1-7. Chapman and Hall, New York (1991).

14. J. Muller. Bot. Rev. 47, 1-142 (1981).

15. R. A. Berner and D. E. Canfield. American J. Science 289, 333-361 (1989).

16. G. Dahlgren. Bot. J. Linn. Soc. 100, 197-203 (1989).

17. M. Eigen and R. Winkler-Oswatitsch. Steps Towards Life, A Perspective on Evolution, p. 122, Oxford University Press, Oxford (1992). 